\title{
Parameterisation of coronal heating: spatial distribution and observable consequences
}

\author{
T. van Wettum ${ }^{1,2}$, S. Bingert ${ }^{1}$, and H. Peter ${ }^{1}$ \\ 1 Max-Planck-Institut für Sonnensystemforschung, 37191 Katlenburg-Lindau, Germany \\ e-mail: vanwettum@mps.mpg.de \\ 2 Institut für Astrophysik, Universität Göttingen, Friedrich-Hund-Platz 1, 37077 Göttingen, Germany
}

Received 14 February 2013 / Accepted 6 March 2013

\section{ABSTRACT}

\begin{abstract}
Aims. We investigate the difference in the spatial distribution of the energy input for parameterisations of different mechanisms to heat the corona of the Sun and possible impacts on the coronal emission.

Methods. We use a 3D magneto-hydrodynamic (MHD) model of a solar active region as a reference and compare the Ohmic-type heating in this model to parameterisations for alternating current (AC) and direct current (DC) heating models; in particular, we use Alfvén wave and MHD turbulence heating. We extract the quantities needed for these two parameterisations from the reference model and investigate the spatial distribution of the heat input in all three cases, globally and along individual field lines. To study differences in the resulting coronal emission, we employ 1D loop models with a prescribed heat input based on the heating rate we extracted along a bundle of field lines.

Results. On average, all heating implementations show a rough drop of the heating rate with height. This also holds for individual field lines. While all mechanisms show a concentration of the energy input towards the low parts of the atmosphere, for individual field lines the concentration towards the foot points is much stronger for the DC mechanisms than for the Alfvén wave AC case. In contrast, the AC model gives a stronger concentration of the emission towards the foot points. This is because the more homogeneous distribution of the energy input leads to higher coronal temperatures and a more extended transition region.

Conclusions. The significant difference in the concentration of the heat input towards the foot points for the AC and DC mechanisms and the pointed difference in the spatial distribution of the coronal emission for these cases show that the two mechanisms should be discriminable by observations. Before drawing final conclusions, these parameterisations should be implemented in new 3D models in a more self-consistent way.
\end{abstract}

Key words. Sun: corona - stars: coronae - magnetohydrodynamics (MHD) - methods: numerical

\section{Introduction}

The question of heating the corona to temperatures well in excess of the photosphere is not yet fully answered, despite several decades of research. For quite a while, two categories based on magnetic mechanisms have been favoured, alternating current (AC) and direct current (DC) heating. In the former case, the magnetic field is driven faster than the Alfvén crossing time, giving rise to magneto-acoustic waves. In the latter, the driving is more gradual and leads to a braiding of field lines. In both cases, the induced (alternating or direct) currents are dissipated and thus heat the coronal plasma in the end. There is ample observational evidence for the presence of Alfvénic waves in the corona that could lead to AC heating (e.g. Tomczyk et al. 2007). Recently, there have been claims that braiding of field lines has been observed (Cirtain et al. 2013), which would lead to DC heating. This would confirm the original theoretical braiding concept by Parker (1972) or the flux-tube tectonics model (Priest et al. 2002). However, the actual microscopic dissipation process is still not answered by any of these concepts.

According to classical transport theory, the actual dissipation should happen on very small scales of a meter and below, which is because of the low value of the magnetic diffusivity (e.g. Boyd $\&$ Sanderson 2003). It is clear that such small scales cannot be reached in a model that is built to describe actually observed solar structures, e.g. an active region that encompasses about $100 \mathrm{Mm}$ in each spatial dimension. Accounting for the spatial complexity of the corona, one needs to employ a 3D model, which then limits the possible spatial resolution in the models to approximately $10 \mathrm{~km}$ to $100 \mathrm{~km}$ (depending on the size of the computational domain). This is a bit better than what can be achieved with current observations, where the spatial resolution in the corona is mostly limited to about $1 \mathrm{Mm}$. Therefore, to understand the appearance of the corona on the observable scales, one has to employ more or less well-founded parameterisations for the spatio-temporal distribution of the heat input. If different parameterisation, e.g. for $\mathrm{AC}$ and $\mathrm{DC}$ heating, result in coronae that look different, a comparison to observations can be employed to test which of the parameterisations fits better. This is then (indirect) confirmation which heating mechanism is dominant in the solar structure under investigation.

In this investigation we use a 3D magneto-hydrodynamics (MHD) model of the corona to study the spatial distribution of the energy input to heat the corona for different parameterisations. In particular, we apply the AC mechanism of energy input through Alfvén waves (van Ballegooijen et al. 2011) and the DC mechanism of MHD turbulence (Rappazzo et al. 2008). As a reference, we use a model that is based on the concept of coronal heating through field-line braiding, as suggested by Parker (1972), where magnetic field lines are braided 
through the granular motion in the photosphere. The currents induced by this process are then dissipated through Ohmic dissipation. The feasibility of maintaining a hot loop-dominated corona through this mechanism has been demonstrated by Gudiksen \& Nordlund (2002, 2005a,b). Since then it has been shown that the transition region and coronal emission synthesized from such models match well to actual observations in a statistical sense (Peter et al. 2004, 2006). In particular, this type of model provides a good understanding of the transition region red-shifts (Zacharias et al. 2011b) and the coronal blue-shifts (Hansteen et al. 2010), even though the interpretation of these models differs. Furthermore, these models provide detailed information on the distribution of the heat input in space, time, and energy (Bingert \& Peter 2011; Bingert \& Peter 2013), produce cool structures that are ejected into the corona (Zacharias et al. 2011a), and give an explanation for the constant cross-section of extreme UV loops (Peter \& Bingert 2012). Therefore we can consider these type of coronal models realistic in the sense that they reproduce major features that we see in actual observations. The main goal of this study is to investigate to what extent the results depend on the assumed form of the Ohmic heating, or if other (parametrized) types of heating would produce similar results.

In Sect. 2 we give an introduction to the 3D MHD model we use and discuss how we parametrize the different mechanisms for the heating. We then discuss the general spatial distribution of the heating rate averaged and along individual field lines (Sect. 3) before we turn to a comparison of the different heating mechanisms (Sect.4). Finally, we present some simple 1D loop models to investigate the observational consequences for the different mechanisms in Sect. 5.

\section{Models of atmospheric structure and analysis}

\subsection{Full $3 D M H D$ with Ohmic heating}

In this investigation we employ the 3D MHD simulation (Bingert \& Peter 2011), this numerical experiment uses the Pencil Code (Brandenburg \& Dobler 2002) to solve the timedependent MHD equations in three dimensions. The computational domain encompasses the volume from the photosphere to the corona of a small active region. The computational domain stretches $50 \times 50 \mathrm{Mm}^{2}$ in the horizontal direction and $30 \mathrm{Mm}$ in the vertical direction and is covered by a $128^{3}$ grid. In the horizontal direction the box is periodic. At the bottom boundary the evolution of the magnetic field in the simulation is driven through a horizontal driver, which has the same properties as the horizontal granular motions (size, velocities, lifetime, power spectrum). The model solves the full energy equation, which includes, in addition to Ohmic heating, optically thin radiative losses (Cook et al. 1989) and, in particular, Spitzer heat conduction parallel to the magnetic field (Spitzer 1962). The latter is pivotal to obtain the proper pressure of the corona set self-consistently. The full set-up has been discussed in detail by Bingert \& Peter (2011).

The Ohmic heating rate (per volume, e.g. measured in $\mathrm{W} / \mathrm{m}^{3}$ ) through the dissipation of currents that are induced by foot point motions is given by

$Q_{\mathrm{Ohm}}=\eta \mu_{0} \dot{j}^{2}$

where $\mu_{0}$ is the magnetic permeability in vacuum and the current $\boldsymbol{j}$ is given by the curl of the magnetic field, $\nabla \times \boldsymbol{B} / \mu_{0}$. The value of the magnetic resistivity is set to $\eta=10^{10} \mathrm{~m}^{2} / \mathrm{s}$, to ensure that the magnetic Reynolds number (when using the grid spacing as a length scale) is of order unity, as discussed in some detail by Bingert \& Peter (2011). As a result the currents will be dissipated at the grid spacing.

After some initialization, the model corona reaches a quasistationary state, i.e. the corona continues to change its thermal structure and harbours strong flows, but the quantities remain comparably constant in a statistical sense (when horizontally averaging over the box). In this state the radiative losses and the heat conduction balance the heat input by Ohmic dissipation and, to a lesser extent, viscous heating. Therefore, the average vertical profiles of temperature and pressure of the corona are mainly determined by the energy inserted into the corona through the Poynting flux. The magnetic diffusivity determines how efficient and at which scales the energy is deposited. For the purpose of this study, we therefore consider $\eta \mu_{0} \boldsymbol{j}^{2}$ as a parameterisation of Ohmic heating.

\subsection{Reduced MHD models for other heating mechanisms}

We compare this parameterisation of Ohmic heating with two parameterisations of coronal heating suggested recently. Both of these suggestions are based on work done with reduced MHD models, where a high resolution can be reached by reducing the equations to only consider the deviations from a (steady) background. Both models solve the heating along a coronal loop and are driven by photospheric motions at the boundaries. As a trade-off for the high resolutions reached by these simulations, concessions are made with regard to certain physical aspects, such as gravity, conduction, or even the presence of a temperature in the energy equation. Therefore they do not provide a possibility to compare the results directly with observables, i.e. through synthesized coronal emission or Doppler shifts. For this one would have to include in particular the heat conduction to allow the coronal pressure to self-consistently adjust to the energy input.

These two heating parameterisations, which are further investigated in this paper, are based on heating through Alfvén wave dissipation and MHD turbulence.

Alfvén waves: this mechanism operates through dissipation of Alfvén waves high in the corona, which are excited in the photosphere. In their model van Ballegooijen et al. (2011) encompass a gravitationally stratified flux tube, with a velocity driver at the bottom boundary that excites the Alfvén waves. These propagate upwards into the corona and and are dissipated there. The resulting loss of magnetic energy is then equated to the energy input into the corona. Based on their model results, van Ballegooijen et al. (2011) derived the following parameterisation (their Eq. (63)) of heating rate per volume:

$Q_{\text {alf }} \propto B^{0.55} L^{-0.92} v_{\text {rms }}^{1.65}$,

where $v_{\mathrm{rms}}$ is the (root-mean-squared) velocity of the foot-point motions, $L$ the length of the magnetic field line (or loop), and $B$ the local magnetic field strength. We will refer to this parameterisation as "Alfvénic heating".

MHD turbulence: this heating mechanism acts through an anisotropic turbulent cascade forming thin elongated current sheets. In their reduced MHD model, Rappazzo et al. (2008) analysed the energy input through this process and derived a parameterisation (their Eq. (68)). One of their exponents in the parameterisation depends weakly on the Alfvén speed, $(\alpha+1) /(\alpha+2)$ ranges from 0.6 to 0.9 for Alfvén speeds from $50 \mathrm{~km} \mathrm{~s}^{-1}$ to $1000 \mathrm{~km} \mathrm{~s}^{-1}$. For the following we thus adopted 
a value of 0.75 . Then one can rewrite the parameterisation of Rappazzo et al. (2008) as

$Q_{\text {turb }} \propto B^{1.75} L^{-1.75} \rho^{0.125} v_{\mathrm{rms}}^{1.25} \ell^{0.75}$,

where $\rho$ is the local density of the plasma and the other parameters are as above. As also suggested by Rappazzo et al. (2008), we assume the injection length $\ell$, i.e. the scale of granules, to be constant. We refer to this as "turbulent heating".

\subsection{Implementation of the heating parameterisations}

We use these parameterisations, Eqs. (1) to (3), to investigate the spatial distribution of heat input into magnetic loops in the corona. For this we extract the relevant parameters from the 3D MHD model along magnetic field lines.

The local magnetic field strength $B$, the mass density $\rho$, and the currents $\boldsymbol{j}=\nabla \times \boldsymbol{B} / \mu_{0}$ can be easily calculated at each grid point of the 3D MHD model. To determine the length $L$ of the field line passing through each grid point and the rms-velocity $v_{\text {rms }}$ at the foot points of these field lines, we trace the field line crossing each grid point in the computational domain. We consider only closed field lines, i.e. those for which the tracing from the grid point in both directions ends up at the bottom boundary. For the parameterisation we use the average of $v_{\text {rms }}$ at both foot points. After the calculation of the trajectories of the field lines, we interpolate $B, \rho$ and $j^{2}$ along each of the field lines. Through this we can investigate the distribution of the heat input for the parameterisations (1) to (3) along individual field lines without running a new simulation with a different heating term in the energy equation (see Sect. 3.2).

The case of the Ohmic dissipation (1) will be self-consistent because the 3D MHD model we employ to extract $Q_{\mathrm{Ohm}}$ uses exactly this form of the heating. Consequently, the other two cases will not be fully self-consistent. However, for the Alfvénic heating $Q_{\text {alf }}(2)$, which depends only on magnetic quantities (field strength and field line length), this should not be a significant problem, at least not in the coronal part of the computational domain, which is what we are interested in. Here the plasma- $\beta$ is much smaller than unity and thus the magnetic field dominates the plasma. Consequently, the heat input will change only the thermal structure, but not the magnetic structure (or only to a small extent), there is no significant back-coupling from the heating rate to the magnetic structure. The situation is less favourable for the turbulent heating $Q_{\text {turb }}(3)$, which also depends on the thermal structure through the mass density. However, the dependence $\left(\rho^{0.125}\right)$ is only weak, which is why this inconsistency should be mostly acceptable. We come back to this issue in Sect.4.2. A set-up with a fully self-consistent treatment of the different heating parameterisations in the framework of new 3D MHD models is planned for future work.

\section{Spatial distribution of the heat input}

\subsection{Horizontal averages}

Using the method described above, we calculate the heating rate for all three parameterisations, Eqs. (1) to (3), at every grid point in the simulation box. Subsequently, we determine the horizontally averaged heating rates (as a function of height), as shown in Fig. 1. Only for Ohmic heating do we use physical units; the turbulent and Alfvénic heating rates are plotted in arbitrary units.

All three parameterisations drop roughly exponentially in the coronal part of the volume. For the Ohmic heating case, this is

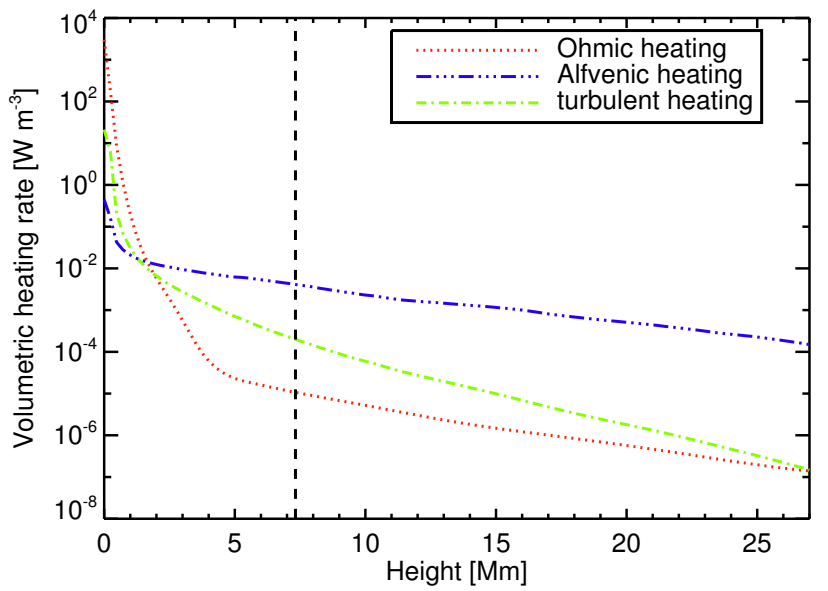

Fig. 1. Horizontally averaged heating rates for different parameterisations. The dashed line indicates the average height of the base of the corona $(\log T[K]=5.5)$. The Alfvénic and turbulent heating rates are in arbitrary units. See Sect. 3.1.

well known from previous studies. The fact that this exponential drop is common to all these three processes underlines the result (in part based on observations) that the energy input into the corona should be concentrated towards the foot points (e.g. Aschwanden et al. 2007). While the (exponential) scale height for the drop of the heating rate is about $5 \mathrm{Mm}$ for the Ohmic and Alfvénic heating, it is only $3 \mathrm{Mm}$ for the turbulent heating. Thus the turbulent heating drops slightly faster than the two other mechanisms.

This common exponential drop for the three mechanisms is interesting because different (1D loop) models made different assumptions about the spatial distribution. While many models have assumed this exponentially dropping heat input (e.g. Serio et al. 1981; Müller et al. 2003), there are also numerous models that assume a spatially constant heating rate (e.g. Patsourakos \& Klimchuk 2006; Klimchuk 2006).

Based on the horizontal averages alone as shown in Fig. 1, one cannot conclude that the distribution of the heat input along each magnetic field line is non-constant, but drops with height. Since the heating rates depend inversely on the loop length, it could be the result of stronger heating along the short field lines in the lower regions, even if the heating rate along each individual field line is constant. In the following we investigate individual field lines to determine if the drop of the horizontally averaged heating rate is because the heating drops with height for each field line or because longer field lines are heated less.

\subsection{Variation along individual field lines}

We make two selections of field lines based on their maximum temperature and density along the loop. Other selections were investigated, but the principle results did not significantly differ from these two selections; thus we limit the discussion to only these two. The first selection of field lines is roughly cospatial with a bright loop in synthesized coronal emission that was studied in detail by Peter \& Bingert (2012). These field lines have lengths between 45 and $50 \mathrm{Mm}$, a maximum temperature exceeding $\log T[\mathrm{~K}]=6.15$, and a minimum density larger than $10^{8.5}$ particles per $\mathrm{cm}^{3}$. We refer to this set as the "bright loop". Figure 2 shows an illustration of the computational domain with the bottom boundary magnetic field indicated. The bright loop is plotted here as the dense cluster of red lines. 


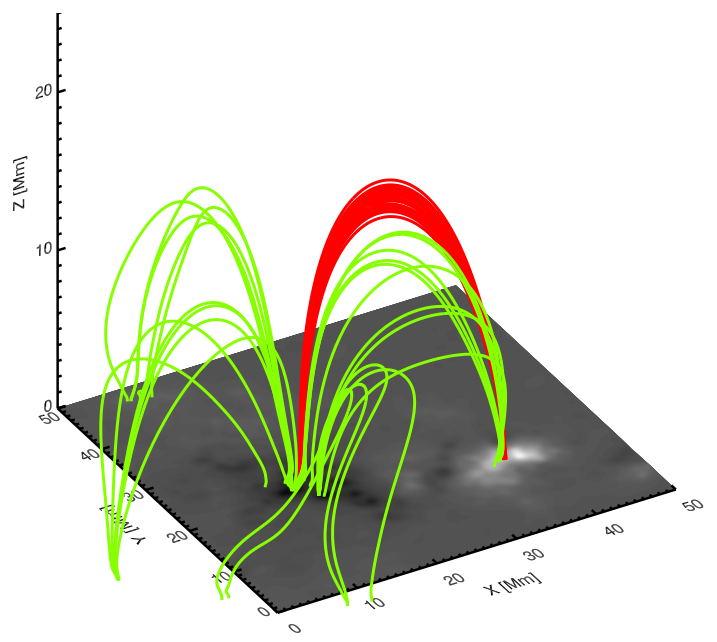

Fig. 2. Three-dimensional illustration of selected field lines for this investigation. The red lines belong to the bright loop selection, and the green ones to selection of the random set of field lines. The gray-scale image shows the magnetogram at the bottom boundary of the simulation (which is based on observations).

The second selection includes field lines which are not limited to the central bright loop but are more randomly distributed throughout the corona. The requirements for the density are similar to those of the bright loop, but has a lower maximum temperature, $\log T /[\mathrm{K}]=6.05$. The lengths of the field lines of this selection range from 40 to $45 \mathrm{Mm}$. This more random selection of field lines provides a test to confirm whether the heating profiles calculated along the bright loop set are typical heating rates. We refer to this set as the "random set". It is drawn in Fig. 2 as the green lines. A subset of 25 randomly selected lines from both selections, has been used for the plots in this paper in order not to clutter the panels.

The results of the calculated heating rates along each of the loops in these subsets are plotted in Figs. 3 and 5. It is clear that the heating rate drops for each field line, irrespective of the heating mechanism we considered here (we discuss the differences between the mechanisms in the following section). This shows that the horizontally averaged heating rates shown in Fig. 1 are not an artefact of averaging over different loops with different lengths, while the heat input is constant along each loop. Also, if we test different sets of loops (or for that matter, all loops reaching up into the corona) we find that the heat input drops strongly with height along each field line. This does not fully rule out that some loops might be heated constantly in space, but we do not find this, at least for the mechanisms checked here.

\section{Comparison of the heating mechanisms}

\subsection{Magnetic field lines in a bright loop}

To compare the parameterisations for Ohmic, Alfvénic, and turbulent heating, we first investigate the set of field lines associated with a bright loop as defined in Sect. 3.2 and shown in red in Fig. 2. The volumetric heating rates for the three parameterisations along individual field lines in the bright loop are shown in Fig. 3. We plot this as a function of the arc length along the field line, where the length of each field line is normalized to unity. The field lines in the set differ in length by $10 \%$ at most.

The most striking difference is that the Ohmic heating varies much more strongly than the Alfvénic and turbulent heating on small scales (smaller than a couple of $\%$ of the field-line length). This is because Ohmic heating depends on the spatial derivatives
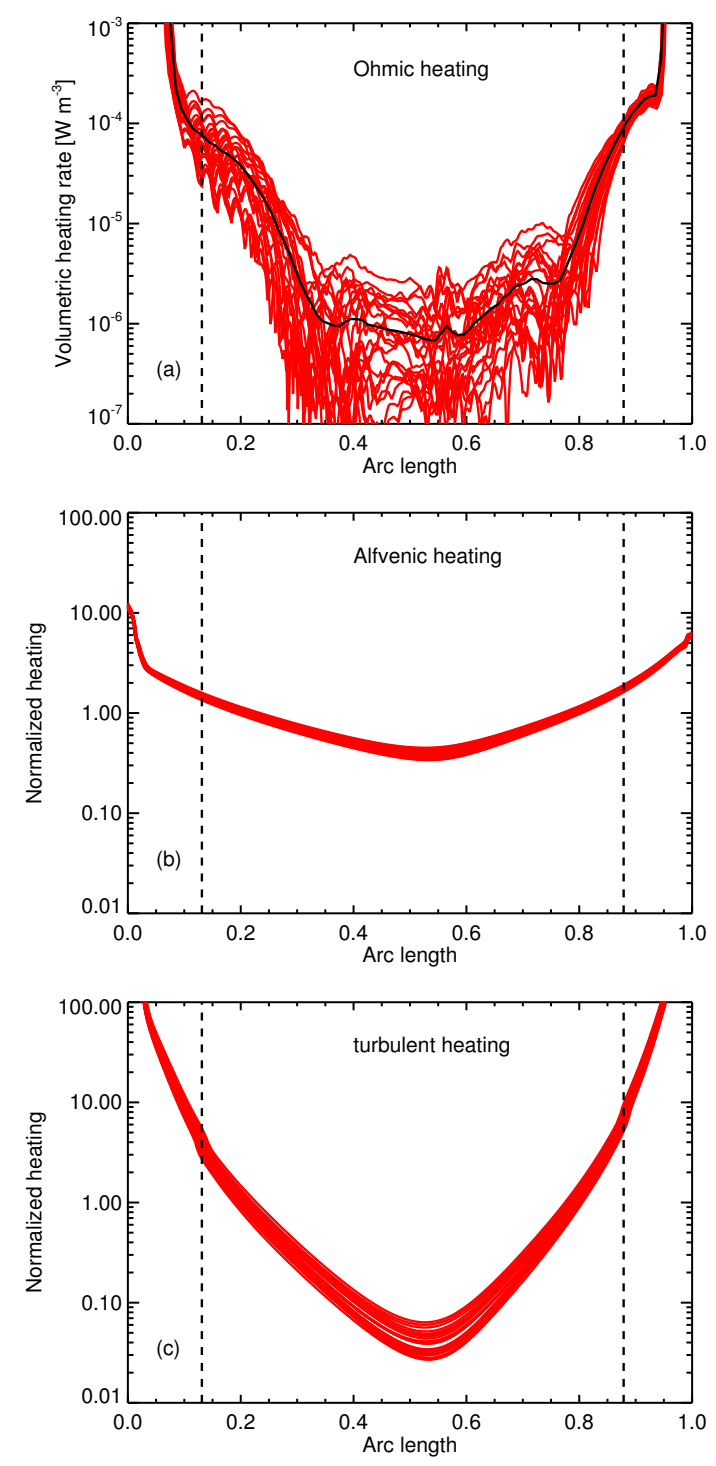

Fig. 3. Volumetric heating rates along individual field lines of the bright loop set, marked in red in Fig. 2. The lengths of the field lines are normalized to unity. The thick black line in the top panel shows the average of the Ohmic heating for the selected field lines. The Ohmic heating rates are averaged over five minutes to reduce the effect of transient events. The dashed lines indicate the average position of the coronal base at $\log T[\mathrm{~K}]=5.5$ for the selected field lines. The Alfvénic and the turbulent heating are plotted normalized to the heat input just below the coronal base. All panels cover the heating rate over four orders of magnitude on the ordinate. See Sects. 3.2 and 4.1.

of the magnetic field (actually, the square thereof). Naturally, these show much stronger small-scale (but well-resolved) variations than the magnetic field itself. The original spatial variation of the Alfvénic and turbulent heating rate in the respective numerical models (van Ballegooijen et al. 2011; Rappazzo et al. 2008) also shows a stronger spatial variation. The heating rate becomes smooth only when the average behaviour of deriving the parameterisations as a function of $B$ and other quantities is considered.

Apart from the small-scale variation, the (smoothed) Ohmic heating and the turbulent heating along the magnetic field lines are rather similar. In both cases the heating rate drops from the base of the corona (indicated in Fig. 3 by vertical dashed lines) to the loop apex by about a factor of 150 to 200 . This is not too 

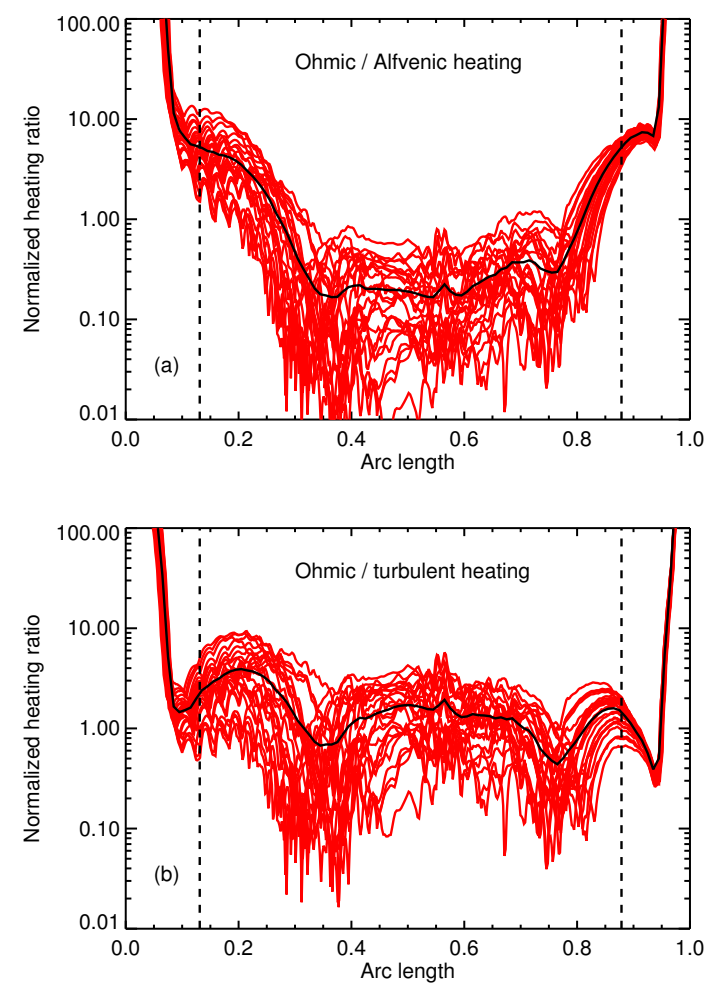

Fig. 4. Normalized ratio of Ohmic heating to Alfvénic and turbulent heating (volumetric heating rates) for the field lines of the bright loop set, marked in red in Fig. 2. The lengths of the field lines are normalized to unity. The thick black lines show the average of the ratios for the selected field lines. The dashed lines indicate the average position of the coronal base at $\log T[\mathrm{~K}]=5.5$ for the selected field lines. Both panels show the ratio over a range of four orders of magnitude. See Sect. 4.1.

surprising because the Rappazzo et al. (2008) 3D reduced MHD model for the turbulent heating is, in principle, quite similar to our 3D MHD model for Ohmic heating (Bingert \& Peter 2011). In both cases the foot points are smoothly driven at boundaries, which braids the magnetic field and induces currents. The reduced MHD model lacks the realistic set-up and the proper treatment of the energy equation to get the coronal pressure correct, but it can afford a much higher resolution in the numerical experiment and properly resolves the turbulent nature of the dissipation process. It is reassuring that these two models provide results for the heating rate that are not too different.

In contrast, the results for the Alfvénic heating following the van Ballegooijen et al. (2011) parameterisation show a different drop of the heating rate. From the coronal base to the top of the loop, the heating rate drops only by a factor of five to six (middle panel of Fig. 3). Comparing the Alfvénic and turbulent parameterisations (2) and (3) clearly indicates that the magnetic field $B$ makes the difference. The lengths $L$ of the field lines in the set are the same within $10 \%$, the horizontal velocities at the foot points $v_{\text {rms }}$ due to the granulation cover only a small range, and the drop of the density $\rho$ is not very important because of the comparably large barometric scale height (and the turbulent heating depends only weakly on $\rho$ ). However, the drop of the magnetic field from the coronal base to the loop apex by a factor of about 20 is mainly responsible for the large drop of the turbulent heating $\left(20^{1.75} \approx 190\right)$ and the only small drop of the Alfvénic heating $\left(20^{0.55} \approx 5\right)$.

To highlight the differences in the spatial distribution of the three heating parameterisations, we plot the ratio of Ohmic to
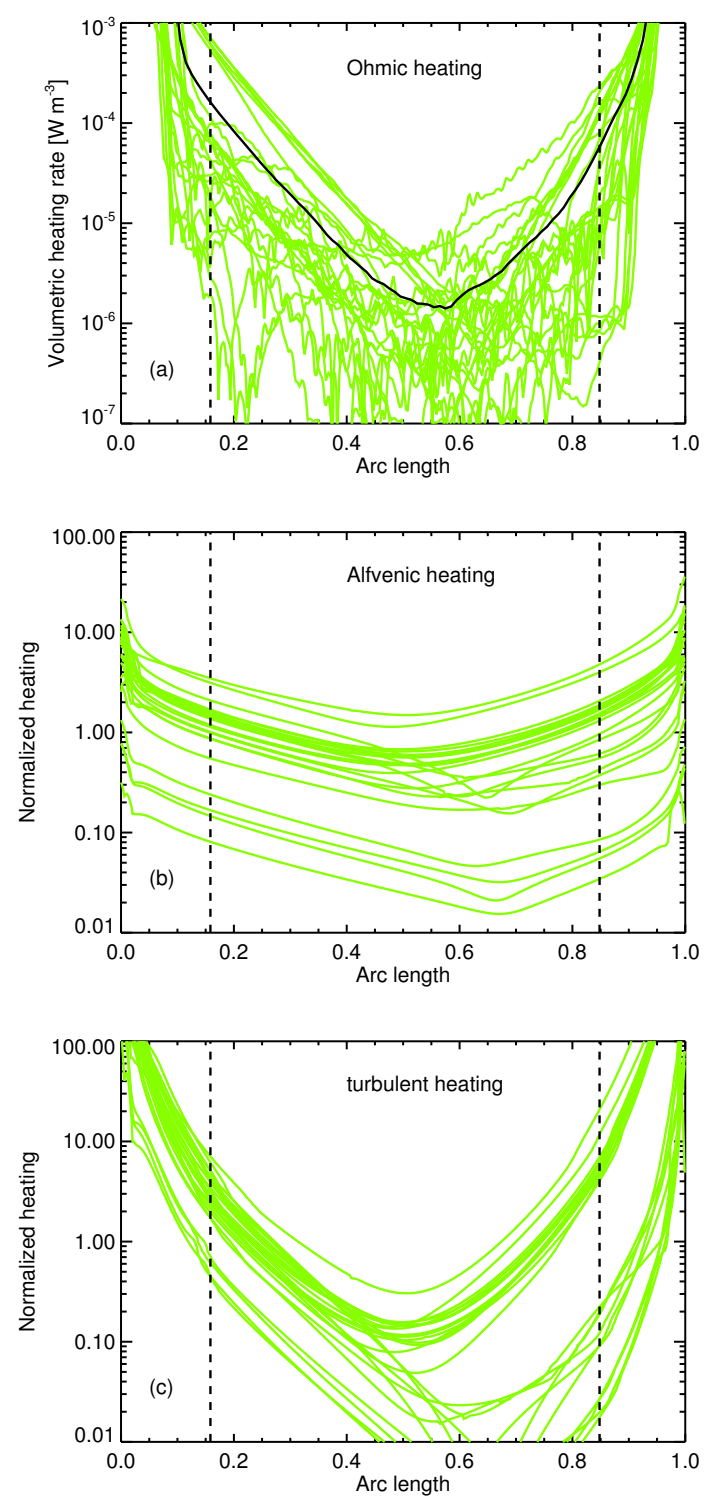

Fig. 5. Similar to Fig. 3, but for the volumetric heating rates of the random set of field lines marked in Fig. 2 as green lines. The asymmetry of the field line shapes shows up clearly, especially for turbulent heating. See Sect. 4.2.

Alfvénic and turbulent heating in Fig. 4. This underlines that (on average) the turbulent heating is quite similar to the Ohmic heating. They both show a much stronger concentration towards the foot points, which is mainly because of the different dependence on the magnetic field strength. The heating rates also differ quite significantly below the base of the corona. This is not surprising because the parameterisations for the Alfvénic and turbulent heating are derived for the corona. So taking them seriously in the chromosphere would be overstretching these approximations. The 3D MHD model with the Ohmic heating shows a much stronger energy input in the chromosphere, which is because of the strong shearing of the magnetic field in the lower, denser part of the atmosphere, where plasma- $\beta$ is no longer smaller than unity.

\subsection{The "random set" of field lines}

The preceding discussion is for a quite special structure, namely, for field lines associated with a bright loop. As a sort of blind test 

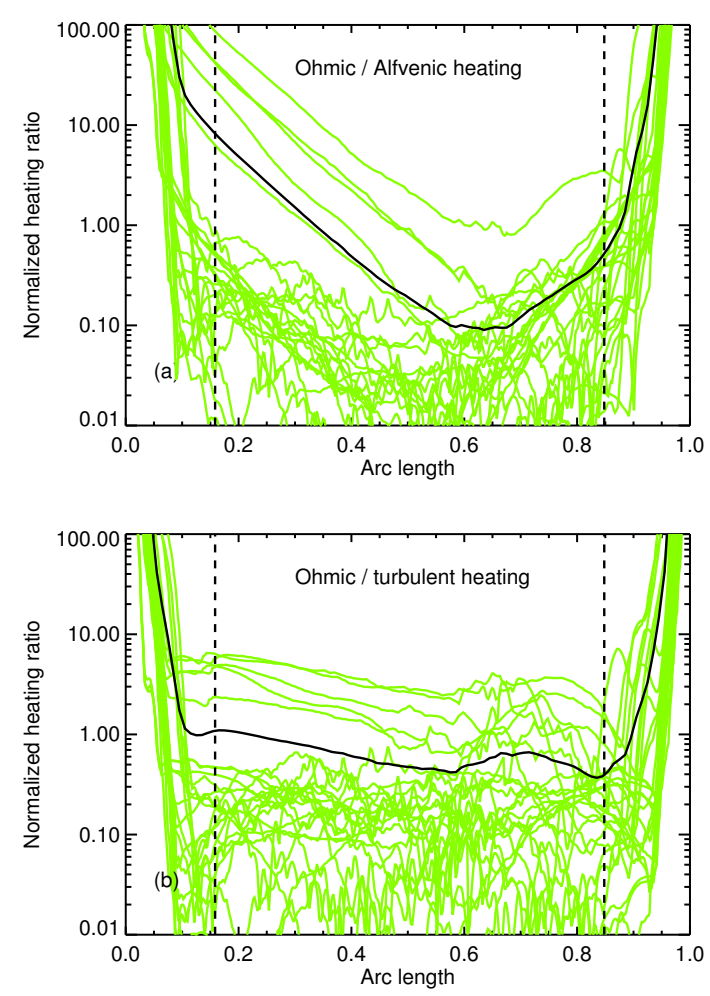

Fig. 6. Similar to Fig. 4, but for the ratios of the heating rates of the random set of field lines marked in Fig. 2 as green lines. See Sect. 4.2.

we now investigate a more random set of field lines that are not associated with any particular coronal structures. This random set is plotted in green in Fig. 2 (see Sect. 3.2 for the definition). The volumetric heating rates and the ratio of the heating rates are plotted in Figs. 5 and 6

Interestingly, this selection gives overall similar results as the bright loop set, albeit with a larger scatter. This is a result of greater variety of field lines that sample different regions in the simulation box in different states. Despite the larger scatter, this clearly shows that the results outlined for the bright loop set can be generalized for the whole corona. This is not too surprising because in a low- $\beta$ plasma the magnetic effects should be not too sensitive to the loading of the field lines with plasma. Thus field lines that are strongly loaded with hot plasma will show the same properties of the (magnetic) heating as other field lines that are not loaded with plasma. Of course, there is still the correlation between heating and coronal density that determines which field lines are loaded with how much plasma (for a discussion of the appearance of loops see e.g. Peter \& Bingert 2012).

There is one pointed difference between the bright loop and the random set, though. The heating rates of the latter show stronger asymmetries between both sides of the loop, which are particularly strong for the turbulent heating parameterisation (middle panel of Fig. 5). This is because some of the field lines in the random set are quite far from being semi-circular (cf. green lines in Fig. 2). These more strangely shaped field lines are hosting the asymmetric heating mainly because of the field-strength asymmetry. Moreover, in the side where the field line does not reach very high into the corona, the density is higher and thus according to Eq. (3) the turbulent heating is stronger. This shows that the back-reaction of the heat input on the magnetic structure cannot be completely neglected (as noted in Sect. 2.3).

\subsection{From field lines to loop models}

We have looked at the spatial distribution of three different parameterisations of coronal heating for two different selections of coronal field lines. The volumetric heating rates of all parameterisations drop for all field lines, and this drop is roughly exponential with height. The main difference is that the heat input for the Ohmic and turbulent case is much more concentrated towards the foot points than in the case of Alfvénic heating.

It is instructive to explore whether this difference in footpoint dominated and more uniform heating has a significant effect on the coronal emission (and the dynamics). Therefore, we now synthesize coronal emission from 1D loop models with a spatial distribution of the heat input similar to the average of the set of field lines associated with the bright loop. Based on this, we can investigate to what extent one can distinguish the heating parameterisations based on observable quantities. Obviously, this can only be a first step because in the end it has to be done within the framework of a 3D MHD model.

\section{One-dimensional coronal loop models}

In the following, we construct simple 1D models of a coronal loop with constant cross-section and a prescribed heating function. All quantities depend only on the arc length along the magnetic field line defining the loop. The velocity is parallel to the loop. Besides accounting for the conservation of mass and momentum (including gravity), we solve the energy equation. The latter accounts for optically thin radiative losses (following Cook et al. 1989) and heat conduction parallel to the magnetic field. This ensures that the coronal pressure is set self-consistently, which is pivotal if the resulting coronal emission radiated from the loop is to be synthesized, as we do here. The 1D models are run using the Pencil Code (Brandenburg \& Dobler 2002) and follow the procedure of Peter et al. (2012).

For the purpose of comparing the synthesized emission from the $1 \mathrm{D}$, loop we adopt the average heating rate of the set of field lines associated with the bright loop (red lines in Fig. 2, definition in Sect. 3.2, discussion in Sect. 4.1). This loop has a height of roughly $15 \mathrm{Mm}$ and a foot-point distance of about $28 \mathrm{Mm}$. This corresponds to a roughly semi-circular shape with a length of about $45 \mathrm{Mm}$ which we will use in our numerical 1D model.

The volumetric heating rate $Q_{i}$ in the $1 \mathrm{D}$ model, which we will assume to fall off exponentially, is

$Q_{i}=H_{0, i} \exp \left(-\frac{z}{\lambda_{i}}\right)$

where $z$ denotes the geometric height and $H_{0, i}$ is the heating rate at $z=0$. The scale height $\lambda_{i}$ for the heating remains to be determined for the three heating parameterisations, here represented by the index $i$.

To determine the scale heights $\lambda_{i}$, we show in Fig. 7 the volumetric heating rates as a function of the geometric height $z$, including both loop legs, and not as a function of the arc length as before. We now fitted a simple exponential function in the form of Eq. (4), which provides values for the $\lambda_{i}$. For the fitting procedure we ignored all data points below the coronal base (at $\log T[\mathrm{~K}]=5.5$, roughly at $3 \mathrm{Mm}$ ). These fits are over-plotted in Fig. 7 and the values for the $\lambda_{i}$ are given. The exponential drop gives quite a good fit to the average variation. As suspected from the discussion in Sect.4.1, Ohmic and turbulent heating show very similar results. This is why in the following we only compare the Ohmic and Alfvénic parameterisations. In the Ohmic case we adopt a scale height of $\lambda_{\mathrm{Ohm}}=1.8 \mathrm{Mm}$, for the Alfvénic 

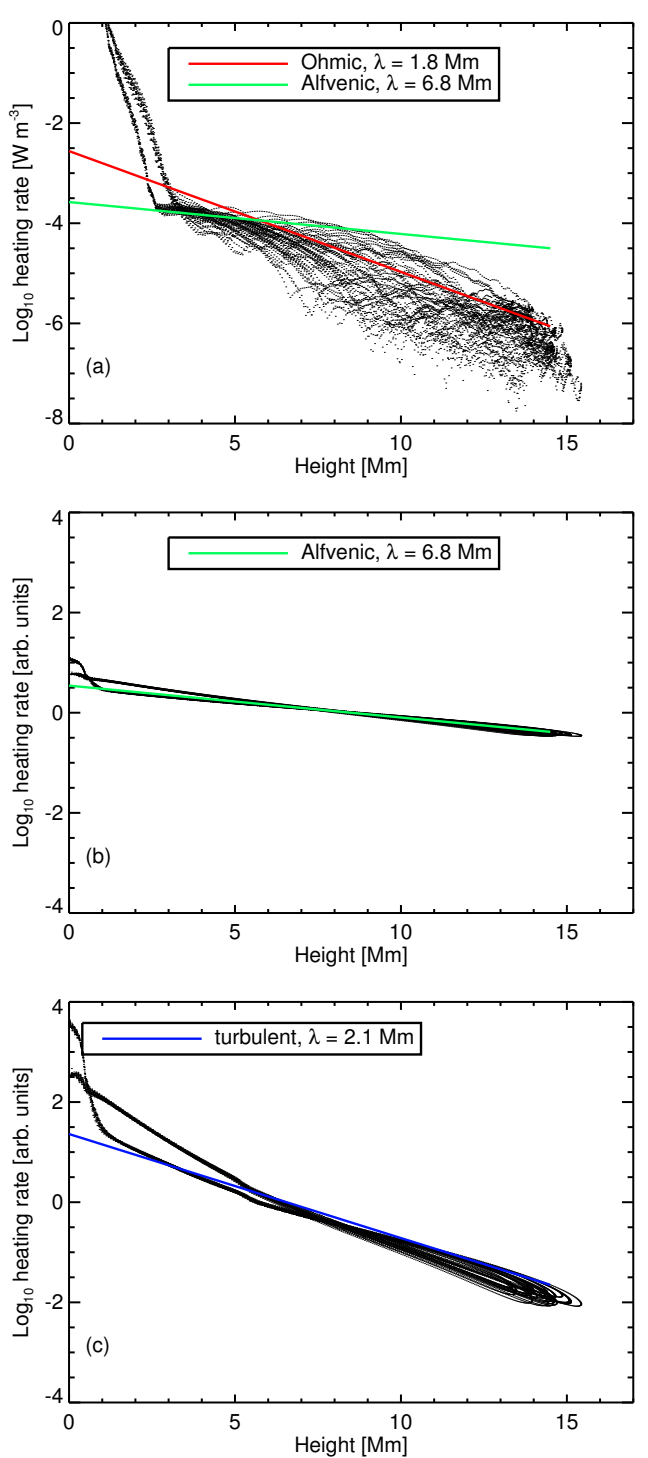

Fig. 7. Volumetric heating rates over geometrical height for the bright loop (red in Fig. 2) for the three parameterisations. The black dots show the actual heating rates along the field lines. The straight lines display the best exponential fit according to Eq. (4). The resulting scale height for the heating rate, $\lambda_{i}$, is listed. For comparison the exponential drop of the Alfvénic heating is over-plotted (in arbitrary units). See Sect. 5.

case $\lambda_{\text {alf }}=6.8 \mathrm{Mm}$. For the Ohmic case we use a heating rate at $z=0$ of about $H_{0, \mathrm{Ohm}} \approx 3 \mathrm{~mW} / \mathrm{m}^{3}$ (see top panel of Fig. 7). We determine the value for $H_{0 \text {,alf }}$ using the requirement that the heat input into the corona (i.e. integrated above the coronal base) has to be the same in both cases. A more detailed description of the results of the 1D models are given in Appendix A.

Because of the short scale height $\lambda_{\mathrm{Ohm}}$, the loop for the parameterisation of the Ohmic heating is subject to a loss of equilibrium near the apex. This process is well documented in the literature (e.g. Müller et al. 2003; Karpen et al. 2006; Peter et al. 2012, and references therein). It leads to the episodic formation of condensations in the loop that eventually slide down into the photosphere. For the following discussion we thus investigate a snapshot in the comparably long time between two condensations (near $t \approx 7500 \mathrm{~s}$, see Appendix A). The loop model of the Alfvénic heating with the longer scale height $\lambda_{\text {alf }}$ reaches a static solution, which we then select for further analysis. For the times we analyse the two 1D models, the velocities along the loop are very close to zero, less than $3 \%$ of the sound speed, in both cases.

The temperature and density along the 1D model loops are plotted in Fig. 8 (top row). In the case of the Ohmic parameterisation (in red), the temperature is below the temperature at the apex as found in the 3D model. The condensation that formed earlier on is an effective sink for the energy, and thus preventing strong condensation, e.g., by a siphon flow induced by asymmetric heating (see Appendix A), could allow the temperature to reach higher values near the apex. However, the focus of the following discussion is not to what extent the $1 \mathrm{D}$ models can reproduce the results of the $3 \mathrm{D}$ model, but if one can find observable differences for the two loop models with different heating parameterisations.

For the Ohmic and the Alfvénic heating we now calculate the coronal emission from the 1D loop model as it would be observed by the extreme UV imager on-board the Solar Dynamics Observatory, the Atmospheric Imaging Assembly (AIA, Lemen et al. 2012). In particular, following the procedure of (Peter et al. 2012), we synthesize the emission for the $131 \AA$ and $171 \AA$ channels that are dominated by emission from Fe VIII and Fe IX from plasma at temperatures of about 5.7 and 5.9 in $\log T$ [K] (Boerner et al. 2012). The resulting emission in these two pass bands for the two loop models is shown in the bottom panel of Fig. 8.

Overall, the (relative) spatial distribution of the $171 \AA$ A emission is quite similar for both, the Ohmic and the Alfvénic heating (Fig. 8 c,d). However, because of a lower density (and temperature), the absolute level of the emission is different. Still, when investigating actual observations to test which heating mechanism might be dominant, we would have to rely mostly on the relative distribution of the emission along the loop and not so much on the absolute level. Thus, at least in the case that we look at here, it would be hard to distinguish the heating mechanisms based on the $171 \AA$ band alone.

A clearer difference is seen in the $131 \AA$ channel, where Alfvénic heating has two distinct "horns" near the foot points of the loop but is significantly weaker in the centre of the loop, when compared to the case of Ohmic heating. This is somewhat unexpected because the Ohmic heating shows a much stronger concentration towards the foot points. However, the emission we see does not directly reflect the spatial variation of the heat input but is a convolution of temperature and density, both of which are set by the heat input. Because of the higher apex temperature for the Alfvénic heating case, the spatial regions where the (comparatively cool) $131 \AA$ emission originates are narrower and shifted down when compared to the Ohmic case.

Depending on the band pass of coronal emission, two quite different heating mechanisms might produce similar or very different spatial distribution of the emission along the loop. In this example the $171 \AA$ band is similar, the $131 \AA$ band is different. For other 1D loop experiments with different total heat input, one can expect to find similar results, even though other channels might then be similar or different. So after all, observations of the coronal emission should hold the potential to distinguish between different spatial distributions of the heat input if one is not focusing on a single emission line or extreme UV bandpass alone.

\section{Conclusions}

In this paper we investigated differences and similarities of three mechanisms to heat the corona: Ohmic heating following 

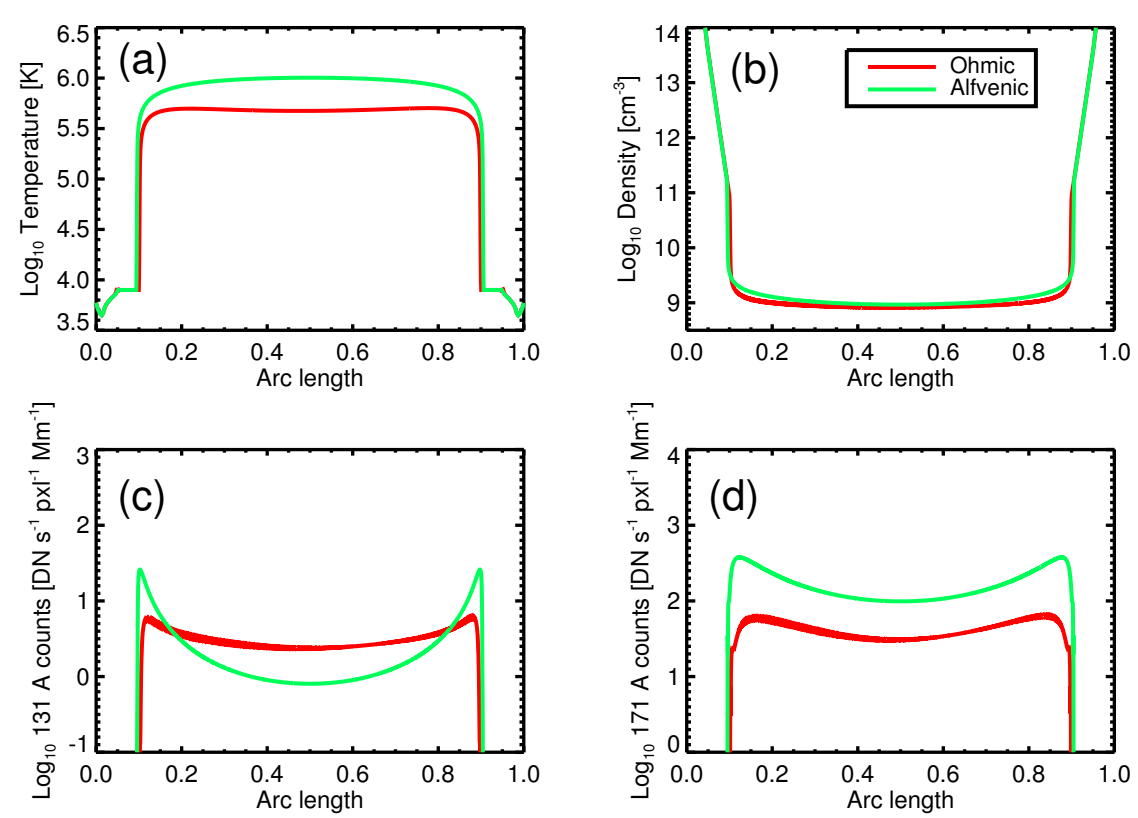

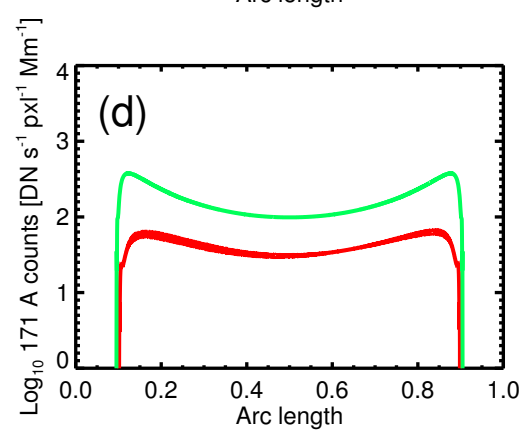

Fig. 8. Variation along the loops in the 1D models for the parameterisation of the Ohmic (red) and Alfvénic heating (green). The arc length is normalized to the loop length of $45 \mathrm{Mm}$. The top panels show the temperature and the density, the velocities are very close to zero. The bottom panels show the coronal emission synthesized from the model as it would be observed with AIA in the $131 \AA$ band c) and the $171 \AA$ band d). See Sect. 5 . braiding of magnetic field lines by photospheric motions, the dissipation of Alfvén waves, and MHD turbulence. For our study we used the results of a self-consistent 3D MHD simulation (Bingert \& Peter 2011). From this we calculated the Ohmic heating rate as resulting from that model, and heating rates that would be given by parameterisations for Alfvénic heating (van Ballegooijen et al. 2011), and turbulent heating (Rappazzo et al. 2008).

We find that all the horizontally averaged heating rates drop roughly exponentially with height. This is true for the average and also when investigating the heating rate along individual field lines. We find a drop of the volumetric heating rate that is roughly exponential with height, along magnetic field lines that are associated with a bright coronal loop in the 3D model and along basically all other field lines reaching into the corona.

The Ohmic and the turbulent heating show roughly the same spatial distribution. This is not really surprising because the reduced MHD model for the turbulent heating is based upon Rappazzo et al. (2008), and is similar in principle to the full 3D MHD model (Bingert \& Peter 2011). In both models the field lines are braided and the non-linearity of the MHD equations drives the formation of small scales. This induces currents at small scales that are dissipated. While the full MHD model properly includes the energy equation, the reduced MHD models allow a much higher resolution. It is reassuring that these two models, using different approaches, give roughly the same result on the spatial distribution of the heating. In contrast, the Alfvénic heating (van Ballegooijen et al. 2011) shows a significantly smaller degree of concentration of the heating rate towards the foot points.

Using the spatial distribution of the heat input from the 3D models, we ran 1D loop models to make a first estimate whether one can distinguish the different mechanisms by the distribution of the coronal emission along the loop. For this we synthesized the emission to how it would be seen with AIA. Here we find that some bands (for our example at $171 \AA$ ) look quite similar, while others (here at $131 \AA$ ) show quite different variations along the loop.
The good news is that the different heating mechanisms will produce different observables (when considering enough bands). However, the bad news is that probably fiddling around with 1D models might be not sufficient because there are too many free parameters. Here we showed only results for one loop for two AIA bands, and the situation is quite different for other loops and/or other bands. Accounting for spatial complexity, new 3D models with a self-consistent treatment of the heat input based on driving in the photosphere will help in pinpointing the observational similarities and differences of the different heating mechanisms.

Acknowledgements. This work was supported by the International Max Planck Research School (IMPRS) on Physical Processes in the Solar System and Beyond. We thank the referee for the constructive feedback and comments.

\section{Appendix A: Details of 1D coronal models}

The 1D loop model describes a semi-circular 1D coronal loop with a length of $45 \mathrm{Mm}$. Along the loop we solve the mass and momentum balance, including gravity. The energy balance includes heat conduction parallel to the magnetic field and optically thin radiative losses. The heating is exponentially dropping according to Eq. (4) with a scale height $\lambda_{i}$. Using the Pencil Code (Brandenburg \& Dobler 2002) we solve these equations on a 2048 grid, closely following Peter et al. (2012). Starting from an initial condition with a prescribed temperature profile in hydrostatic equilibrium, we evolve the equations.

The results of the numerical experiments are shown in Fig. A. 1 for the parameterisation of the Ohmic heating $\left(\lambda_{\mathrm{Ohm}}=\right.$ $1.8 \mathrm{Mm})$ and in Fig. A.2 for the Alfvénic heating $\left(\lambda_{\mathrm{alf}}=\right.$ $6.8 \mathrm{Mm})$. Temperature, density, and velocity are plotted as a space-time plot, i.e. as a function of arc length along the loop (normalized to the loop length) and time.

In the case of Alfvénic heating (Fig. A.2), the coronal loop reaches a stable static state after some 2000 s. For the discussion of this parameterisation in Sect. 5, we thus select a snapshot at $t=2500 \mathrm{~s}$, where the velocity is very close to zero, less than $3 \%$ of the sound speed. 

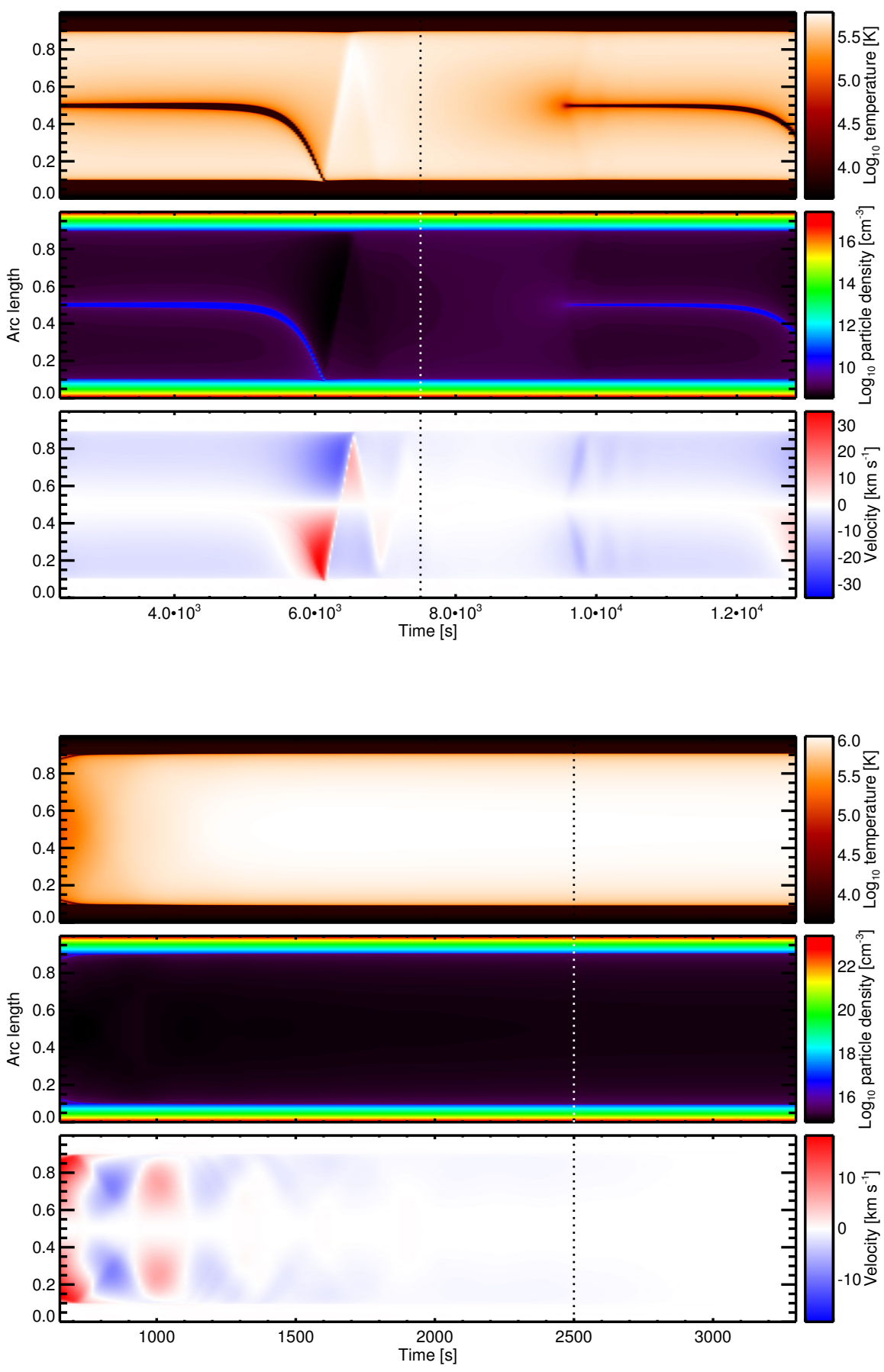

Fig. A.1. Variation of temperature, density, and velocity along the loop in the $1 \mathrm{D}$ model. Here we show the results for the parameterisation of Ohmic heating with a scale height of $\lambda_{\mathrm{Ohm}}=1.8 \mathrm{Mm}$ for an exponentially dropping heat input following Eq. (4). The arc length is normalized to the loop length of $45 \mathrm{Mm}$. The vertical dashed line indicates the time of the snapshot, which is further analysed in Sect. 5.

Fig. A.2. Same as Fig. A.1, but for the case of Alfvénic heating with a scale height of $\lambda_{\text {alf }}=6.8 \mathrm{Mm}$.
The situation is different for the Ohmic heating (Fig. A.1). The strong concentration of the heating towards the foot points evaporates chromospheric material, which then climbs up the loop towards the apex, where the heating rate is quite low. As the density increases, the radiative losses of the plasma increase and the plasma effectively cools. In a runaway process, a condensation forms because radiation becomes more efficient with decreasing temperature. Finally the condensation slides down one side of the loop. Because the heat input is kept constant, the whole procedure starts again. This process of loss of equilibrium is well known (e.g. Müller et al. 2003; Karpen et al. 2006; Peter et al. 2012), and the results we find for the onset of the condensation (depending on $\lambda_{i}$ ) are consistent with previous studies. From an inspection of Fig. A.1, it is clear that there are long stretches of time between condensations wherein the loop is comparably stable at hot temperatures without any condensation present. During the time span shown in Fig. A.1, this covers about $3000 \mathrm{~s}$. This is long compared to the sound-crossing time, which is of the order of $400 \mathrm{~s}$. Arguing that we want to catch the loop undergoing Ohmic heating in a phase when it is hot and free of condensation, we select a snapshot at $t=7500 \mathrm{~s}$ (cf. Fig. A.1) for the further analysis in Sect. 5. At this time the velocity along the loop is almost zero everywhere, so that we consider this a quasi-steady state.

The 3D MHD model our study, based upon Bingert \& Peter (2011), does not find such condensations in their synthesized emission. In that 3D model, the heat input along the loop is not symmetric, which is evident from the top panel of Fig. 3. 
Even comparably small deviations from a symmetric heating will lead to different pressures at the coronal base of the two loop legs, which will drive a siphon flow through the loop (e.g. Boris \& Mariska 1982). To some extent, such a siphon flow will prevent strong condensations from forming because the flow carries away the plasma as soon as it starts to condense (Z. Mikić, priv. comm.). Thus we do not see a condensation in our 3D model. Furthermore, because condensations do not form, the loop apex can reach higher temperatures, as we see in the 3D model.

We see the condensations form in the more idealized 1D loop model with a perfectly symmetric heating rate. However, in view of the above discussion it is reasonable to concentrate on the phase between the condensations and to use the snapshot at $t=$ $7500 \mathrm{~s}$ for the analysis in Sect. 5.

\section{References}

Aschwanden, M. J., Winebarger, A., Tsiklauri, D., \& Peter, H. 2007, ApJ, 659, 1673

Bingert, S., \& Peter, H. 2011, A\&A, 530, A112

Bingert, S., \& Peter, H. 2013, A\&A, 550, A30

Boerner, P. F., Edwards, C. G., Lemen, J. R., et al. 2012, Sol. Phys., 275, 41

Boris, J. P., \& Mariska, J. T. 1982, ApJ, 258, L49

Boyd, T. J. M., \& Sanderson, J. J. 2003, The Physics of Plasmas (Cambridge University Press)
Brandenburg, A., \& Dobler, W. 2002, Comput. Phys. Commun., 147, 471

Cirtain, J. W., Golub, L., Winebarger, A. L., et al. 2013, Nature, 493, 501

Cook, J. W., Cheng, C.-C., Jacobs, V. L., \& Antiochos, S. K. 1989, ApJ, 338 , 1176

Gudiksen, B. V., \& Nordlund, ̊̊. 2002, ApJ, 572, L113

Gudiksen, B. V., \& Nordlund, A. 2005a, ApJ, 618, 1031

Gudiksen, B. V., \& Nordlund, Å. 2005b, ApJ, 618, 1020

Hansteen, V. H., Hara, H., De Pontieu, B., \& Carlsson, M. 2010, ApJ, 718, 1070

Karpen, J. T., Antiochos, S. K., \& Klimchuk, J. A. 2006, ApJ, 637, 531

Klimchuk, J. A. 2006, Sol. Phys., 234, 41

Lemen, J., Title, A. M., Akin, D. J., et al. 2012, Sol. Phys., 275, 14

Müller, D., Hansteen, V. H., \& Peter, H. 2003, A\&A, 411, 605

Parker, E. N. 1972, ApJ, 174, 499

Patsourakos, S., \& Klimchuk, J. A. 2006, ApJ, 647, 1452

Peter, H., \& Bingert, S. 2012, A\&A, 548, A1

Peter, H., Gudiksen, B., \& Nordlund, Å. 2004, ApJ, 617, L85

Peter, H., Gudiksen, B., \& Nordlund, Å. 2006, ApJ, 638, 1086

Peter, H., Bingert, S., \& Kamio, S. 2012, A\&A, 537, A152

Priest, E. R., Heyvaerts, J. F., \& Title, A. M. 2002, ApJ, 576, 533

Rappazzo, A. F., Velli, M., Einaudi, G., \& Dahlburg, R. B. 2008, ApJ, 677, 1348 Serio, S., Peres, G., Vaiana, G. S., Golub, L., \& Rosner, R. 1981, ApJ, 243, 288

Spitzer, L. 1962, Physics of Fully Ionized Gases

Tomczyk, S., McIntosh, S. W., Keil, S. L., et al. 2007, Science, 317, 1192

van Ballegooijen, A. A., Asgari-Targhi, M., Cranmer, S. R., \& DeLuca, E. E. 2011, ApJ, 736, 3

Zacharias, P., Bingert, S., \& Peter, H. 2011a, A\&A, 532, A112

Zacharias, P., Peter, H., \& Bingert, S. 2011b, A\&A, 531, A97 